\title{
Development of the High-resolution FUV Detector for the BepiColombo Mission
}

\author{
By Go MURAKAMI, Kazuo YOSHIOKA, and Ichiro YOSHIKAWA \\ Department of Earth and Planetary Science, Graduate School of Science, The University of Tokyo, Tokyo, Japan
}

(Received May 2nd, 2008)

\begin{abstract}
The PHEBUS (Probing of Hermean Exosphere By Ultraviolet Spectroscopy) instrument on Mercury Planetary Orbiter in the BepiColombo mission is a dual FUV-EUV spectrometer (EUV: $55-155 \mathrm{~nm}$, FUV: $145-315 \mathrm{~nm}$ ). We are now developing the compact detector system sensitive to FUV airglow emissions of the Mercury. The FUV detector is required to have high spatial resolution $(512 \times 512$ pixels) so that the wavelength resolution of the PHEBUS instrument should be 2 $\mathrm{nm}$ at the FUV range. The FUV detector consists of a $\mathrm{Cs}_{2} \mathrm{Te}$ photocathode, microchannel plates (MCPs), and a resistive anode encoder. In a position-sensitive system with a resistive anode encoder, the spatial resolution is determined by the signal-to-noise ratios at the anode terminals. Therefore, a high and stable electron gain of MCPs allows the position determination of each photoelectron event with high spatial resolution. We studied a method for achieving a high and stable electron gain. We fabricated a test model of the FUV detector incorporating a clamped pair of MCPs (V-stack) followed by a gap and a clamped triplet of MCPs (Z-stack) in cascade. We investigated the effect of the negative inter-stack potential on the PHD and the spatial resolution by means of calculation and experiments. As a result, the negative inter-stack potential made the electron gain more stable and the spatial resolution higher by $\sim 14 \%$. In this paper we report the specific performance of the test model of the FUV detector.
\end{abstract}

Key Words: Mercury, BepiColombo, Ultraviolet, Microchannel Plate

\section{Introduction}

Mariner-10 UV measurements and telescopic spectroscopy from the Earth identified six elements $(\mathrm{Ca}$, $\mathrm{Na}, \mathrm{K}, \mathrm{H}, \mathrm{He}$, and $\mathrm{O}$ ) in the Mercury's exosphere. Other species are expected, e.g. $\mathrm{H}_{2}, \mathrm{OH}$, and some noble gasses (Ar, Ne, and $\mathrm{Xe}$ ). All species representative of the surface composition, directly produced by impact vaporization driven by micrometeoroids, physical sputtering, photo-stimulated desorption, and thermal desorption from the regolith, should also be present. To determine the composition of the Mercury's exosphere, the PHEBUS (Probing of Hermean Exosphere By Ultraviolet Spectroscopy) instrument in the BepiColombo mission will measure the emission lines of the exosphere. PHEBUS is a dual FUV-EUV spectrometer working in the wavelength range from 55 to $315 \mathrm{~nm}^{1,2)}$. The each detector of the PHEBUS instrument is a 2-D photon-counting device consisting of microchannel plates (MCPs) and a resistive anode encoder (RAE).

In space applications MCPs are popularly used as photon detectors, especially in the EUV range $e^{3,4,5,6}$. One of the position sensitive anodes for MCPs is a resistive anode encoder (RAE) $)^{7)}$. In the position sensitive detector with an RAE, the ratio of the charge signals delivered at its four corners or edges depends only on the $X$ and $Y$ centroid location of the event, and so the $X-Y$ coordinates of each event can be determined in essentially real time with a pair of ratio circuits fed by these signals. The simplicity both in the anode itself and in the associated electronics has led to the resistive anode becoming one of the most popular readout methods for photon counting systems.

In a resistive anode position-sensitive system, the spatial resolution depends on the signal-to-noise ratio (SNR) at the anode terminals and therefore depends on the MCP gain. Here, "signal" refers to the charge produced by a single photoelectron, and is synonymous with the gain of the electron multiplier structure. The "noise" is mainly caused by the extraneous electrical noise introduced into the position analyzing system and the thermally generated noise. The double (so called "Chevron" or "V-stack") or triple ("Z-stack") MCPs are commonly used as a method of obtaining a high gain $\left(>10^{6}\right)$. Ideally the output pulses should be all the same amplitude for good pulse counting. An MCP stack, however, does not give a certain value of the gain for every photoelectron event. The statistical variation of the gain of a V-stack or a Z-stack MCP is generally characterized by the pulse height distribution (PHD) with typical values of FWHM of about $80-120 \%$. Therefore, it is desirable that the MCP stack exhibits a high and stable gain with a narrow PHD.

The position-sensitive detectors using an RAE and a usual V-stack or Z-stack MCP typically give spatial resolutions of the order of $100 \times 100$ to $250 \times 250$ pixels. Lampton and Carlson $^{7)}$ reported that their sample detector consisting of two MCPs in a V-stack configuration and an RAE achieved the resolution of $100 \times 100$ pixels. Recently, the detector using a Z-stack MCP and an RAE was mounted in the Telescope of Extreme Ultraviolet (TEX) onboard the SELENE satellite ${ }^{8)}$. The detector had an active area of $32 \times 32 \mathrm{~mm}$ and it achieved the $250-\mu \mathrm{m}$ resolution, corresponding to the format of $128 \times 128$ pixels. 
This degree of the spatial resolution has proven satisfactory for a number of applications in laboratory and space flight photon counting. However, in the BepiColombo mission the FUV detector is required to have higher spatial resolution of $512 \times 512$ pixels so that the wavelength resolution of the PHEBUS instrument should be $2 \mathrm{~nm}$ at the FUV range.

One of the techniques to improve the spatial resolution of the detector with an RAE is using five MCPs in a set of V-stack and Z-stack ${ }^{9,10)}$. Firmani et al. ${ }^{9)}$ and Floryan and Johnson $^{10)}$ investigated the performance of the similar type of detector incorporating five MCPs arranged in a set of V- and Z-stacks with an inter-stack gap (a V-Z gap). They each measured the spatial resolution of their own detector under a wide variety of applied potentials, and found that a negative potential applied across the V-Z gap enabled the detector to achieve the spatial resolution of the order of $500 \times 500$ pixels. It was not completely understood, however, why applying the negative potential across the V-Z gap improved the spatial resolution. Firmani et al. ${ }^{9)}$ suggested that applying the negative potential expanded the electron cloud arriving at the Z-stack and a high stable gain was achieved due to a large number of excited channels. On the contrary, Floryan and Johnson $^{10)}$ suggested as follows: (1) electrons with sufficiently high energies to reach the Z-stack were emitted far enough up the channels in the V-stack and highly collimated by the channels, (2) the number of excited channels at the Z-stack was reduced, and (3) the Z-stack MCP was completely operated in the saturated mode to exhibit a narrow PHD.

It is necessary to understand the effect of the negative potential applied across the V-Z gap on the electron distribution at the Z-stack input, in order to use this technique for the BepiColombo mission and to reduce the effort at experimental measurements for optimization purposes. Computer simulation gives us useful information on the distribution of the electron cloud from the MCP output ${ }^{11,12)}$. Zanodvorov et al. ${ }^{13)}$ presented a "ballistic" model of the charge cloud propagation based on the differential electron distributions at the MCP output, which had been measured in the experiments of Bronshteyn et al. ${ }^{14)}$. In this approach, no interactions between the electrons in the cloud are taken into account, i.e., the electric field is assumed to be constant rather than

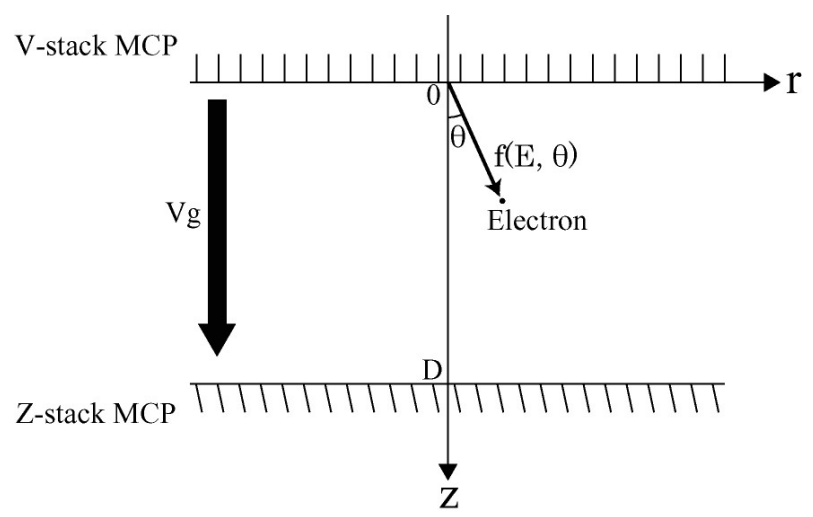

Fig. 1. Schematic diagram of the coordinate. self-consistent.

In this study, we use a similar ballistic approach for calculating the distribution of the electron cloud across the V-Z gap. The result gives us important information on the effect of the negative potential across the V-Z gap. Then, to confirm the result of the calculation, experiments are described to investigate the dependency of the spatial resolutions and the PHDs on the applied negative potential. This work has a direct impact on the way in which MCPs can be optimized to satisfy the requirement for the PHEBUS instrument in the BepiColombo mission.

\section{Calculation}

The statistical nature of the electron avalanche, originating at the output of the MCP channels, inhibits a precise calculation of the charge cloud evolution. The angles and velocities of the output electrons exhibit large variations depending not only on the MCP intrinsic parameters (such as the length-to-diameter ratio $\mathrm{L} / \mathrm{D}$, the secondary electron emission coefficient of the semi-conductive layer, the end spoiling, etc.), but also on the mode and sometimes even the history of operation. However, below we suggest some approximation which enables me to simulate the electron cloud development in the gap between the V-and Z-stacks (V-Z gap) and obtain some information on the size of the electron cloud at the input surface of the Z-stack MCP.

Following the same approach as the past studies ${ }^{11,13)}$, the trajectories of all electrons in the cloud are assumed to be purely ballistic, and the differential distribution function at the output of the MCP, measured by Bronshteyn et al. ${ }^{14)}$, is used. For simplicity, it is assumed further that (1) the original size of the charge cloud at the $\mathrm{V}$-stack output, determined by the number of excited channels $\left(\sim 6.5\right.$ channels $\left.^{15)}\right)$, is negligible, that (2) no interactions occur between the electrons in the cloud, and that (3) the bias angle of the output MCP is $0 \mathrm{deg}$ and then the electron distribution is symmetric to the channel axis. Fig. 1 shows the coordinate system for our model. Using the electron distribution function, it is possible to calculate the ballistic trajectories of all electrons and to estimate the spatial distribution of the cloud at any distance from the MCP output.

We use one of the experimental distributions of Bronshteyn et al. ${ }^{14)}$, which was measured using a single MCP with an L/D of 60:1, 20- $\mu \mathrm{m}$ channels, and an applied voltage of $1.4 \mathrm{kV}$. The electron distribution function at the MCP output can be approximated by the following function $^{13)}$ :

$$
f(E, \theta)=e^{-\tau^{2} / 2 \Delta^{2}}\left|A(\tau) e^{-\left(E-E_{0}\right)^{2} / 2 \sigma_{0}{ }^{2}}-B(\tau) e^{-\left(E-E_{1}(\tau)\right)^{2} / 2 \sigma_{1}^{2}(\tau)}\right|,
$$

where $E$ and $\theta$ are the electron output energy and angle, respectively, and $\tau=\tan \theta$. The unknown parameters in this distribution were chosen to fit the experimental data ${ }^{14)}$ as follows:

$$
\begin{gathered}
E_{0}=3 \mathrm{eV}, \sigma_{0}=12 \mathrm{eV}, \Delta=0.2, \\
A(\tau)=50.398 \mathrm{e}^{7.3822 \tau}, B(\tau)=25.992 \mathrm{e}^{9.8642 \tau}, \\
E_{1}(\tau)=314.45 \mathrm{e}^{-6.9515 \tau}, \sigma_{1}(\tau)=147.62 \mathrm{e}^{-9.7077 \tau} .
\end{gathered}
$$


Energy distribution of electrons at MCP output

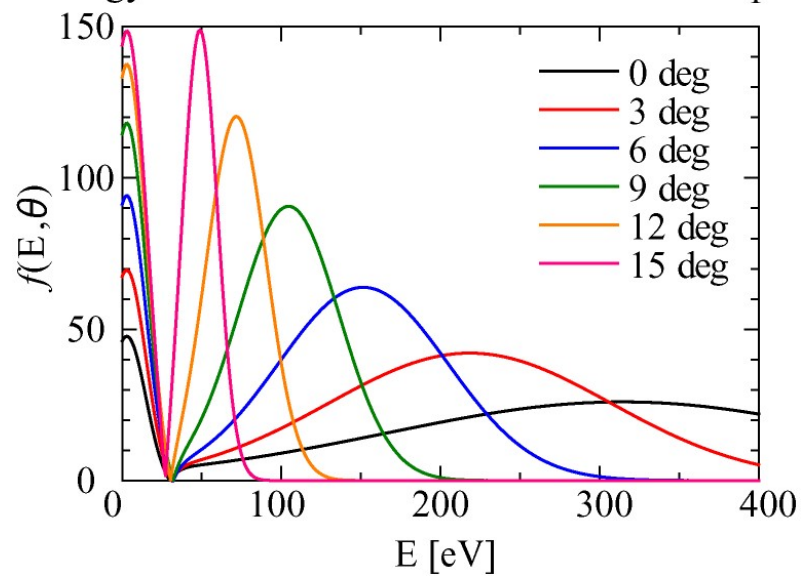

Fig. 2. Energy distribution curves of electrons at MCP output.

Electron distribution at the Z-stack input

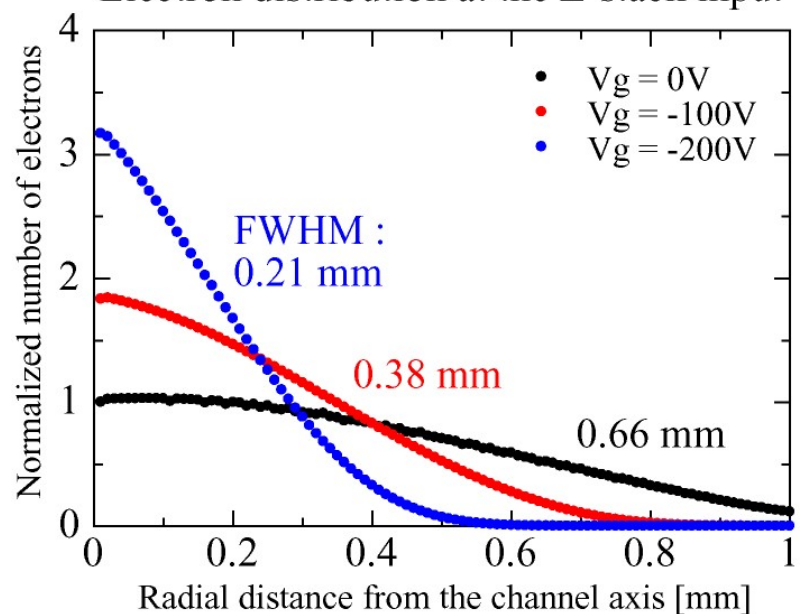

Fig. 3. The distribution of the electron cloud at the Z-stack input.

Fig. 2 shows the distribution function $f(E, \theta)$ at various constant values of $\theta$. The equation of motion of an electron in the cloud at the moment $t$ can be written as,

$$
\begin{gathered}
r=\sqrt{\frac{2 E}{m_{e}}} t \sin \theta, \\
z=\sqrt{\frac{2 E}{m_{e}}} t \cos \theta+\frac{q_{e} V_{g} t^{2}}{2 m_{e} D},
\end{gathered}
$$

where $r$ is the radial distance from the channel axis, $z$ is the distance from the $\mathrm{V}$-stack output, $D$ is the distance between the $\mathrm{V}$ - and Z-stacks, $V_{\mathrm{g}}$ is the accelerating bias in the V-Z gap, and $q_{\mathrm{e}}$ and $m_{\mathrm{e}}$ are the electron charge and mass, respectively. In this work, potentials accelerating electrons from the $\mathrm{V}$-stack toward the Z-stack are considered positive. Using Eq. (3) and Eq. (4), we obtain the radial distance $r_{0}$ at the Z-stack input $(z=D)$.

Using the electron distribution function Eq. (1) (summarized in Fig. 2), we calculated $r_{0}$ of all electrons and estimated the cloud spatial distribution at the input surface of the Z-stack with various values of $V_{\mathrm{g}}$. The radial distributions of electrons, arriving at the Z-stack input with sufficient energy to excite secondary electrons $\left(>10 \mathrm{eV}^{16)}\right)$, are calculated with the parameters of $D=2.5$ $\mathrm{mm}$ and $V_{\mathrm{g}}=0,-100$, and $-200 \mathrm{~V}$. Fig. 3 shows the result. In Fig. 3, the total number of electrons arriving at the $\mathrm{Z}$-stack for each $V_{\mathrm{g}}$ is normalized. Applying the negative inter-stack potential clearly reduced the size of the electron cloud. This means that the outer component of the electron cloud consisting of the low energy electrons emitted at large angles (as shown in Fig. 2) is mainly eliminated. Therefore, the result of the calculation indicates that the negative inter-stack potential allows the Z-stack MCP to be operated in the completely saturated mode and a narrow PHD will be achieved, as suggested by Floryan and Johnson ${ }^{10)}$.

\section{Measurement}

The experimental approach was also performed to check whether the result from the calculation is realistic. The prototype detector used in the measurements consists of an input window made of synthetic silica, a $\mathrm{Cs}_{2} \mathrm{Te}$ photocathode, five MCPs in a set of V-and Z-stacks, and an RAE. The photograph and the schematic geometry of the detector are shown in Fig. 4 and Fig. 5, respectively. The photocathode converts the incoming photons into photoelectrons. The photoelectron is accelerated towards the MCP in the cathode gap. The photoelectrons impact the walls of the MCP channels causing a charge avalanche, giving an overall charge multiplication of $\sim 2 \times 10^{7}$. This

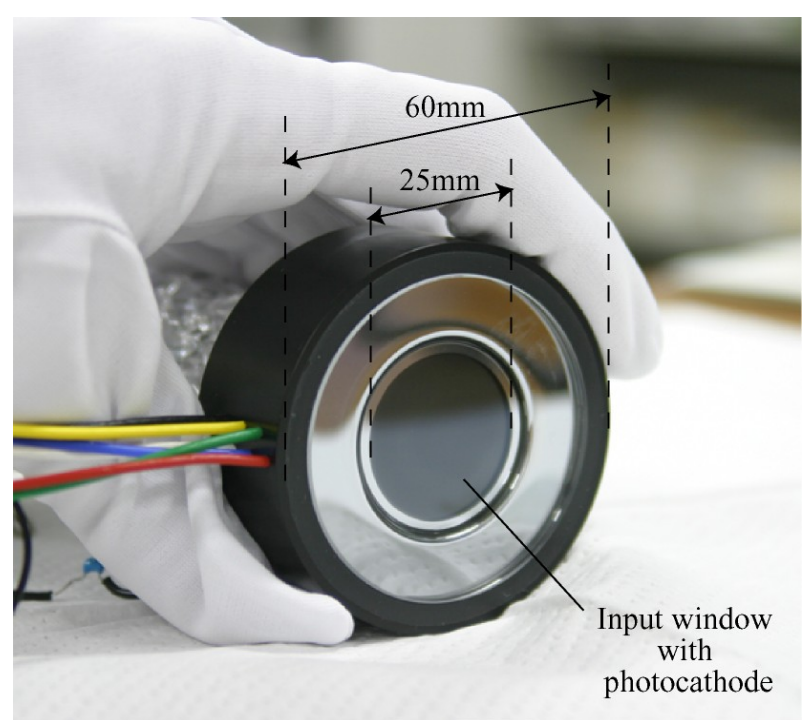

Fig. 4. A photograph of the prototype FUV detector.

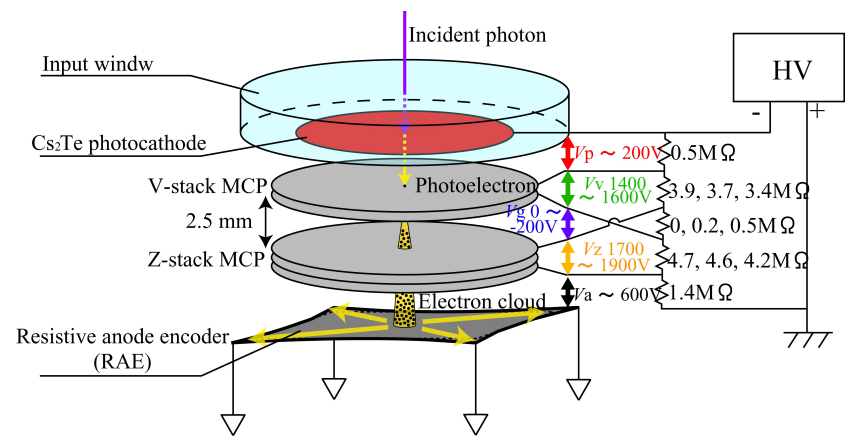

Fig. 5. A schematic diagram of the prototype FUV detector. 


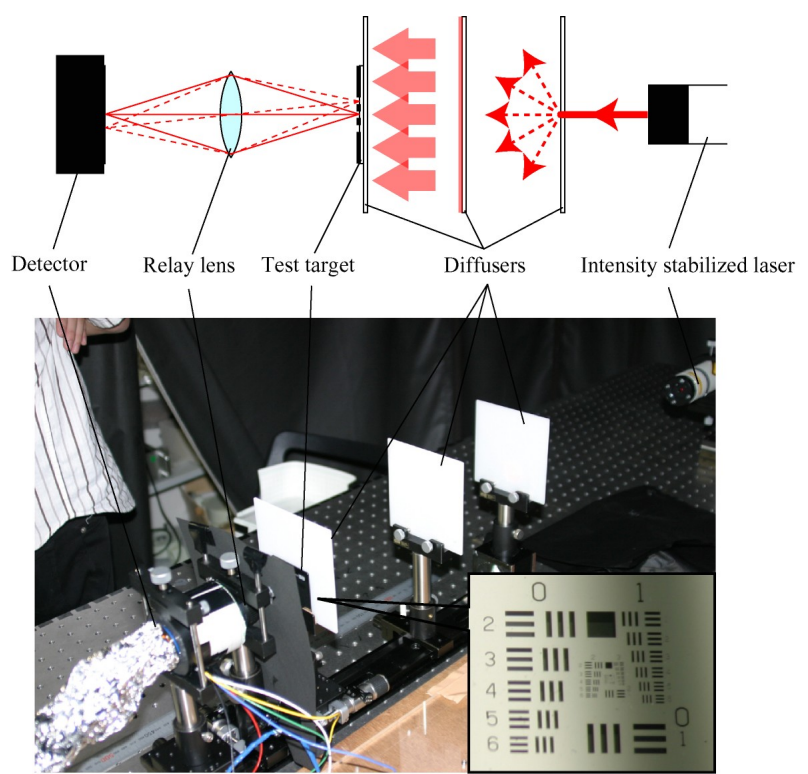

Fig. 6. A schematic diagram (upper) and a photograph (lower) of the setup of the measurement.

charge cloud is drifted from the MCP output to the RAE, and its centroid position can be determined. The four output charge signals from the RAE are amplified and shaped by four preamplifiers. The discriminator discards signals which are too small to be properly processed and signals resulting from simultaneous or near-simultaneous events on the RAE. For valid signals, the centroid position of the electron cloud is calculated by measuring the peak voltage of the pre-amplifiers for each corner A, B, C, and $\mathrm{D}$ of the RAE. The following algorithm is then used to obtain its position in the $\mathrm{X}-\mathrm{Y}$ coordinates:

$$
\begin{aligned}
& X=\frac{A+B}{A+B+C+D}, \\
& Y=\frac{B+C}{A+B+C+D} .
\end{aligned}
$$

The MCPs incorporated in the detector is arranged in a set of V-stack (two plates) and Z-stack (three plates) with a gap of $2.5 \mathrm{~mm}$, as shown in Fig. 5. Each MCP has a circular shape with an active diameter of $25 \mathrm{~mm}$, a bias angle of $12 \mathrm{deg}$, a 10- $\mu \mathrm{m}$ channel diameter, and an L/D (a ratio of length to channel diameter) of $40: 1$. The resistances of the $\mathrm{V}$-stack and the Z-stack are $\sim 90 \mathrm{M} \Omega$ and $\sim 120 \mathrm{M} \Omega$, respectively. The RAE has an active area of $23 \times 23 \mathrm{~mm}^{2}$. In this measurement, however, we use a circular active area of $16-\mathrm{mm}$ diameter defined by an aperture mask placed in contact with the output surface of the Z-stack to avoid the distortion. The detector requires five separate potentials applied as shown in Fig. 5: the potential between the photocathode and the input surface of the $\mathrm{V}$-stack $\left(V_{\mathrm{p}}\right)$, that across the $\mathrm{V}$-stack $\left(V_{\mathrm{v}}\right)$, that between the output surface of the $\mathrm{V}$-stack and the input surface of the Z-stack $\left(V_{\mathrm{g}}\right)$, that across the Z-stack $\left(V_{\mathrm{z}}\right)$, and that between the output surface of the Z-stack and the resistive anode $\left(V_{\mathrm{a}}\right)$. It is possible to operate the detector with a wide variety of combinations of these five potentials. In this work, potentials which accelerate electrons from the photocathode toward the anode are considered positive, and those which decelerate electrons in this direction are considered negative. The applied voltages of $V_{\mathrm{p}}$ and $V_{\mathrm{a}}$ were constantly $\sim 200 \mathrm{~V}$ and $\sim 500 \mathrm{~V}$, respectively, for all the measurements.

We measured the spatial resolution of the detector using the USAF 1951 resolution test target. Our experimental setup is shown schematically in Fig. 6. The light for all tests was $633 \mathrm{~nm}$ light produced by an intensity-stabilized laser. The test target was illuminated uniformly through diffusers, and the image of the test pattern was projected onto the photocathode by a relay lens. Optical focus of the test-pattern image on the photocathode was obtained by taking a series of spatial resolution measurements (described below) at various distances between the relay lens and the detector, and by determining the position where the maximum resolution was achieved. Fig. 7 shows a typical image of the test target and a cross-sectional histogram at $6.3 \mathrm{lp} / \mathrm{mm}$ across it, obtained with $V_{\mathrm{v}}$ of $1420 \mathrm{~V}, V_{\mathrm{g}}$ of $0 \mathrm{~V}$, and $V_{\mathrm{z}}$ of $1710 \mathrm{~V}$ applied. The contrast was calculated from the images of the test target with a simple formula, (Max - Min) / (Max + Min). Analyzing the profiles of the test-pattern images

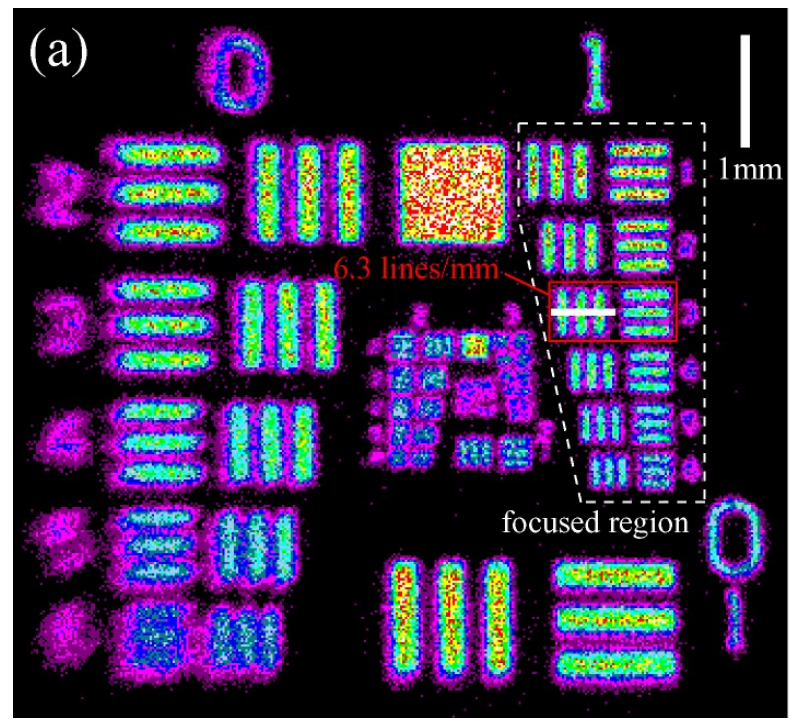

(b)

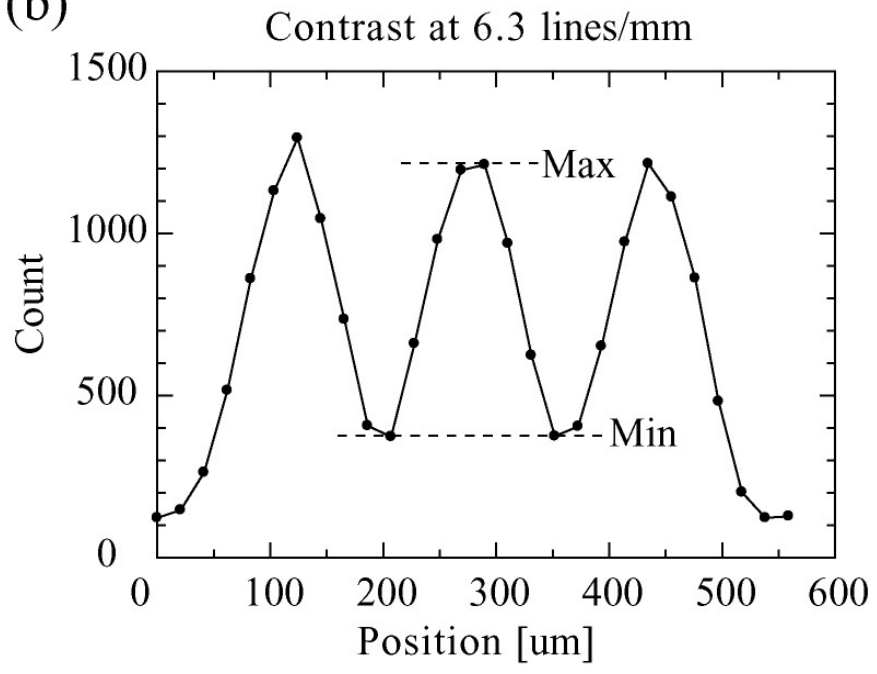

Fig. 7. (a) A test target image obtained by the prototype detector.

(b) A cross section profile of (a) taken along with the white line. 
(a)

Pulse Height Distribution

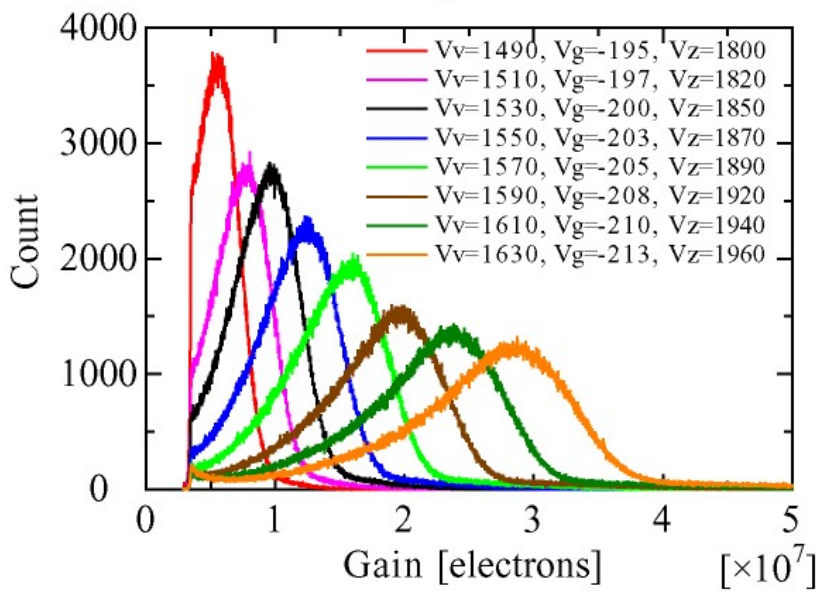

(b)

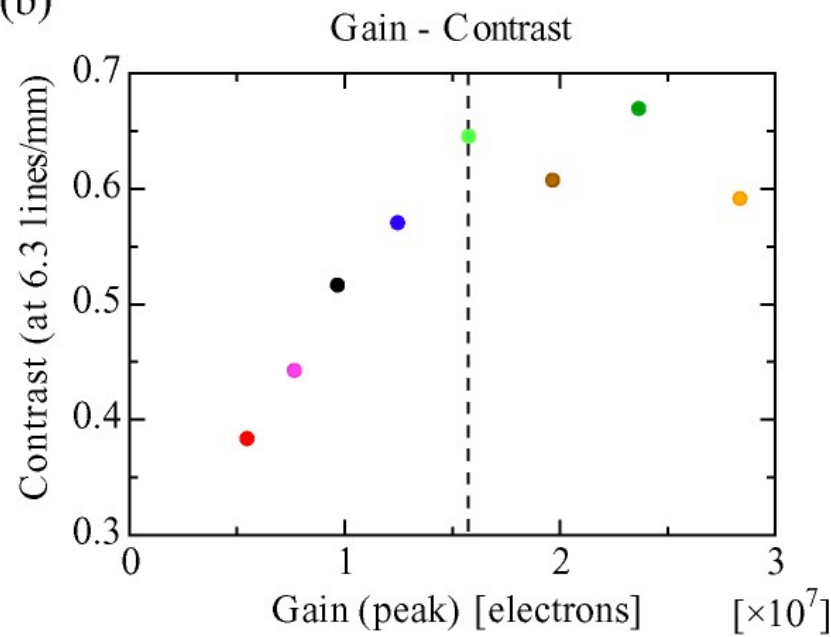

Fig. 8. (a) Pulse height distributions (PHDs) with various high-voltage applied. (b) Dependence of the contrast at 6.3 lines/mm on the gain.

allows the resolution of the detector to be determined.

In this work, we recorded a number of test target images and pulse height distributions obtained with different combinations of applied potentials. We investigated the relationship between the negative inter-stack potential, the spatial resolution, and the pulse height distribution with the experimental approach.

\section{Results and Discussion}

In the first measurement, the dependence of the spatial resolution on the gain and the PHD was examined. We measured the PHDs and spatial resolutions with various potentials applied, and the results are shown in Fig. 8. Each applied potential is also displayed. As seen in Fig. $8 \mathrm{a}$, the peak gain increases and the distribution becomes wider as the applied potential increases. In Fig. 8b, while the contrast linearly increases with the gain below $1.6 \times 10^{7}$, there is no correlation between the contrast and the gain above $1.6 \times 10^{7}$. This result means that the spatial resolution mainly depends on the number of events with low gains $\left(<\sim 0.4 \times 10^{7}\right.$ in this measurement) which have the low SNR. In this measurement, therefore, it was confirmed that the spatial resolution of the detector surely depends on the gain and the PHD of the MCPs.

In the second measurement, the effect of the negative inter-stack potential $\left(V_{\mathrm{g}}\right)$ on the PHD was examined. Fig. 9 shows the PHDs with $V_{\mathrm{g}}$ of $0 \mathrm{~V},-76 \mathrm{~V}$, and $-205 \mathrm{~V}$ applied. The applied potentials across the $\mathrm{V}$-stack $\left(V_{\mathrm{v}}\right)$ and across the Z-stack $\left(V_{\mathrm{z}}\right)$ are controlled appropriately so that each PHD has approximately the same gain at the peak. The PHD clearly got narrower as $V_{\mathrm{g}}$ increased. The full width at half maximum (FWHM) of the PHD (PHR: pulse height ratio) was excellently improved from $165 \%\left(V_{\mathrm{g}}=\right.$ $0 \mathrm{~V})$ to $38 \%\left(V_{\mathrm{g}}=-205 \mathrm{~V}\right)$. This narrowing of the PHD introduced by applying the negative inter-stack potential is consistent with the prediction from our calculation.

Finally, the dependence of the spatial resolution on $V_{\mathrm{g}}$ was examined. Fig. 10 shows modulation transfer functions with $V_{\mathrm{g}}$ of $0 \mathrm{~V},-76 \mathrm{~V}$, and $-205 \mathrm{~V}$ applied. The spatial frequencies where the contrasts equal to 0.33 are also displayed. Applying the negative inter-stack potential improved the spatial resolution by $\sim 14 \%$. This result is consistent with that obtained in the second measurement. In Fig. 10, furthermore, there seems to be no difference between the spatial resolutions with $V_{\mathrm{g}}$ of $-76 \mathrm{~V}$ and $-205 \mathrm{~V}$. This characteristic can be explained by the result of the first measurement, i.e. nearly the same numbers of events with low gains below $\sim 0.4 \times 10^{7}$ were achieved. As

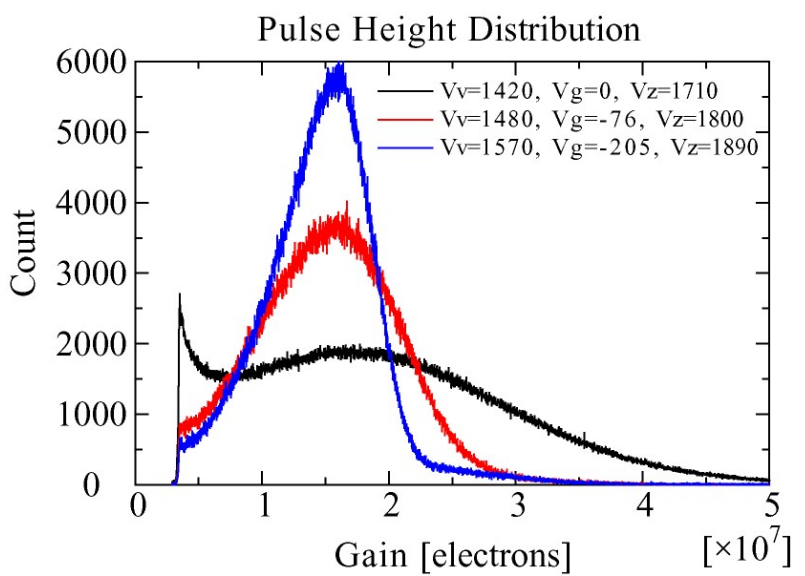

Fig. 9. Dependency of the pulse height distribution on the negative inter-stack potential $\left(V_{\mathrm{g}}\right)$

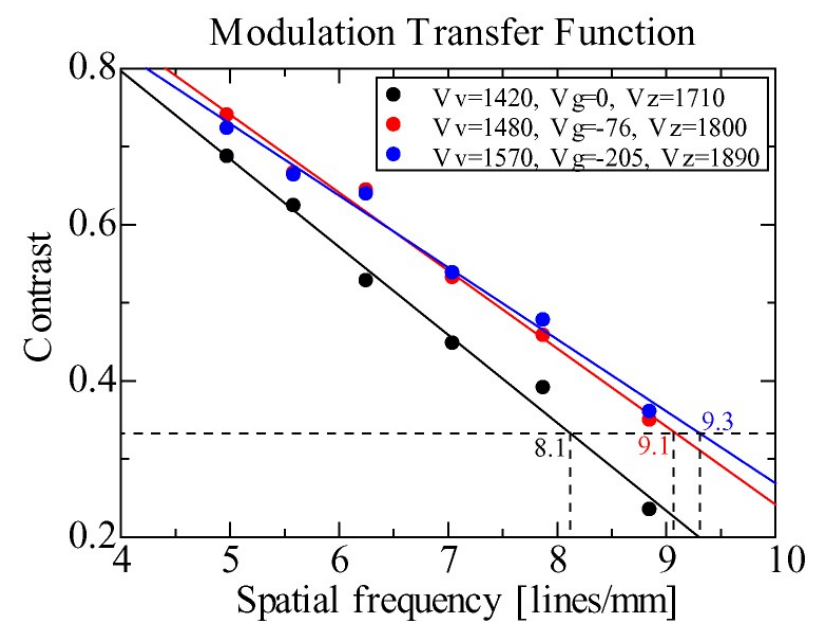

Fig. 10. Dependency of modulation transfer function on $V_{\mathrm{g}}$. 
a result, the spatial resolution of $54 \mu \mathrm{m}$, corresponding to $400 \times 400$ pixels in our detector, was achieved by using $\mathrm{V}$ and Z-stacks and applying the negative inter-stack potential.

The achieved resolution of the prototype FUV detector is lower than that of the order of $500 \times 500$ pixels, required for the PHEBUS instrument, which was achieved by Firmani et al. ${ }^{9)}$ and Floryan and Johnson ${ }^{10)}$. This difference would mainly result from the deficiency of optimizing the potentials applied between the photocathode and the $\mathrm{V}$-stack input $\left(V_{\mathrm{p}}\right)$ and between the Z-stack output and the RAE $\left(V_{\mathrm{a}}\right)$. Floryan and Johnson ${ }^{10)}$ reported that the resolution depends on $V_{\mathrm{a}}$ and especially on $V_{\mathrm{p}}$. They achieved the highest resolution of $500 \times 500$ pixels with $V_{\mathrm{p}}$ of $700 \mathrm{~V}$, while it was $200 \mathrm{~V}$ in our measurement. It is necessary to measure the spatial resolution of the detector with higher $V_{\mathrm{p}}$ for the future missions. Nevertheless, this study revealed the effect of the negative potential applied across the inter-stack gap on the distribution of the electron cloud, the PHD, and the spatial resolution by the calculation and the experiments. Using the same technique we can optimize the characteristics of the position sensitive detector incorporating an RAE to the BepiColombo mission.

\section{Conclusion}

We have developed a test model of the high-resolution FUV detector for the BepiColombo mission. We have investigated the effect of the negative potential applied across the inter-stack (V-Z) gap on the PHD and the spatial resolution by means of calculation and experiments. The calculation with a simple ballistic model showed that the negative inter-stack potential $\left(V_{\mathrm{g}}\right)$ reduced the size of the electron cloud by $\sim 70 \%$. The result suggests that under such a condition the Z-stack MCP is operated in the completely saturated mode and exhibits a narrow PHD. On the other hand, we measured the PHDs and the spatial resolutions of the prototype FUV detector with various potentials applied, and revealed the following points.

(1) The spatial resolution depends on the PHD, especially on the number of the events with low gains. This result is explained by the dependency of the resolution on the SNR of the output pulses.

(2) Applying the negative $V_{\mathrm{g}}$ reduces the width of the PHD from PHR $=165 \%\left(V_{\mathrm{g}}=0 \mathrm{~V}\right)$ to $38 \%\left(V_{\mathrm{g}}=-205 \mathrm{~V}\right)$. This result is consistent with that of the calculation.

(3) Applying the negative $V_{\mathrm{g}}$ improves the spatial resolution by $\sim 14 \%$. This result is explained by the results of (1) and (2).

Finally, the spatial resolution of $54 \mu \mathrm{m}$, corresponding to $400 \times 400$ pixels in our detector, was achieved. We can optimize the technique using five MCPs and an RAE and apply it to the PHEBUS instrument in the BepiColombo mission. Further measurements, however, with a higher potential applied between the Z-stack output and the RAE are necessary to achieve the spatial resolution of the order of $500 \times 500$ pixels required for the PHEBUS instrument.

\section{References}

1) Yoshikawa, I., Yamazaki, A., Murachi, T., Kameda, S., Sagawa, H., Okano, S., Okada, T., and Nakamura, M.: Development of an extreme ultraviolet imaging spectrometer for the BepiColombo mission, Adv. Space Res., 33 (2004), pp.2195-2199.

2) Yoshioka, K., Hikosaka, K., Murakami, G., Yoshikawa, I., Yamazaki, A., and Nozawa, H.,: Development of the EUV detector for the BepiColombo mission, Adv. Space Res., 41 (2007), pp.1392-1396.

3) Yoshikawa, I., Nakamura, M., Hirahara, M., Takizawa, Y., Yamashita, K., Kunieda, H., Yamazaki, T., Misaki, K., and Yamaguchi, A.: Observation of He II emission from the plasmasphere by a newly developed EUV telescope on board sounding rocket S-520-19, J. Geophys. Res., 76 (1997), pp.19897-19902.

4) Nakamura, M., Yoshikawa, I., Yamazaki, A., Shiomi, K., Takizawa, Y., Hirahara, M., Yamashita, K., Saito, Y., and Miyake, W.: Terrestrial plasmaspheric imaging by an extreme ultraviolet scanner on Planet-B, Geophys. Res. Lett., 27(2) (2000), pp.141-144.

5) Yoshikawa, I., Yamazaki, A., Shiomi, K., Yamashita, K., Takizawa, Y., and Nakamura, M.: Photometric measurement of cold helium ions in the magnetotail by an EUV scanner onboard Planet-B: Evidence of the existence of cold plasmas in the near-Earth plasma sheet, Geophys. Res. Lett., 27(21) (2000), pp.3567-3570.

6) Yamazaki, A., Tashiro, S., Nakasaka, Y., Yoshikawa, I., Miyake, W., and Nakamura, M.: Sounding-rocket observation of O II 83.4-nm emission over the polar ionosphere, Geophys. Res. Lett., 29 (2002), pp.2005-2008.

7) Lampton, M., and Carlson, C. W.: Low-distortion resistive anodes for two-dimensional position-sensitive MCP systems, Rev. Sci. Instrum., 50(9) (1979), pp.1093-1097.

8) Yoshikawa, I., Yamazaki, A., Murakami, G., Yoshioka, K., Kameda, S., Ezawa, F., Toyota, T., Miyake, W., Taguchi, M., Kikuchi, M., Nakamura, M., Sakanoi, T., and Okano, S.: Telescope of extreme ultraviolet (TEX) onboard SELENE: science from the Moon, Earth, Planets and Space, 60 (2008), pp.407-416.

9) Firmani, C., Ruiz, E., Carlson, C. W., Lampton, M., and Paresce, F.: High-resolution imaging with a two-dimensional resistive photon counter, Rev. Sci. Instrum., 53(5) (1982), pp.570-574.

10) Floryan, R. F., and Johnson, C. B.: Resistive anode photomultiplier tube optimum operating conditions for photon correlation experiments, Rev. Sci. Instrum., 60(3) (1989), pp.339-343.

11) Tremsin, A. S., and Siegmund, O. H.: Spatial distribution of electron cloud footprints from microchannel plates: Measurements and modeling, Rev. Sci. Instrum., 70(8) (1999), pp.3282-3288.

12) Saito, M., Saito, Y., Asamura, K., and Mukai, T.: Spatial charge cloud size of microchannel plates, Rev. Sci. Instrum., 78 (2007), 023302.

13) Zanodvorov, N. P., Zolina, N. K., Tyutikov, A. M., and Flegontov, Y. A.: Estimation of the modulation-transfer functions of microchannel plates, Radio Eng. Electron. Phys., 25 (1980), pp.129-133.

14) Bronshteyn, I. M., Yevdokimov, A. V., Stozharov, V. M., and Tyutikov, A. M.: Differential secondary-emission characteristics of microchannel plates, Radio Eng. Electron. Phys., 24 (1979), pp.150-152.

15) Wiza, J. L., Henkel, P. R., and Roy, R. L.: Improved microchannel plate performance with a resistive anode encoder, Rev. Sci. Instrum., 48(9) (1977), pp.1217-1218.

16) Siegmund, O. H. W., Vallerga, J., and Wargelin, B.: Background events in microchannel plates, IEEE Trans. Nucl. Sci., 35(1) (1988), pp.524-528. 